\title{
The Effect of Prophylactic Anticoagulation with Heparin on the Brain Cells of Sprague-Dawley Rats in a Cardiopulmonary-Cerebral Resuscitation Model
}

\author{
Wenxun Liu, ${ }^{1,2}$ Yun Wang, ${ }^{2}$ Xiaohong Zhou, ${ }^{2}$ Kerong Hai, ${ }^{2}$ Danting Jia, ${ }^{1}$ \\ and Qingshan $Y e \mathbb{B}^{1,2}$ \\ ${ }^{1}$ Ningxia Medical University, Yinchuan, China \\ ${ }^{2}$ Department of Anesthesiology, People's Hospital of Ningxia Hui Autonomous Region, Yinchuan, China \\ Correspondence should be addressed to Qingshan Ye; bgycyj2005@sina.com
}

Received 16 June 2019; Revised 8 July 2020; Accepted 25 August 2020; Published 11 September 2020

Academic Editor: Min Li

Copyright (C) 2020 Wenxun Liu et al. This is an open access article distributed under the Creative Commons Attribution License, which permits unrestricted use, distribution, and reproduction in any medium, provided the original work is properly cited.

\begin{abstract}
After a cardiac arrest (CA) of 5 to 10 min, a marked activation of blood coagulation occurs and microthrombi are found in the cerebral vessels. These microcirculatory disturbances directly affect the outcome on cardiopulmonary resuscitation (CPR). The purpose of this study was to investigate the effects and potential mechanisms of prophylactic anticoagulation on rat brain cells after cerebral CPR. After setting up an asphyxial CA model, we monitored the basic parameters such as the vitals and survival rate of the rats and assessed the respective neurological deficit (ND) and histological damage (HD) scores of their brain tissues. We, furthermore, investigated the influence of heparin on the expressions of TNF- $\alpha$, IL- $1 \beta$, CD 40 , NF- $\kappa$ B, and HIF- $1 \alpha$ after asphyxial CA. The results showed that anticoagulation with heparin could obviously improve the outcome and prognosis of brain ischemia, including improvement of neurological function recovery and prevention of morphological and immunohistochemical injury on the brain, while significantly increasing the success rate of CPR. Treatment with heparin significantly inhibited the upregulation of CD40, NF- $\kappa$ B, and HIF- $1 \alpha$ induced by asphyxial CA. Thrombolysis treatment may improve the outcome and prognosis of CPR, and future clinical studies need to evaluate the efficacy of early heparin therapy after CA.
\end{abstract}

\section{Introduction}

Cardiac arrest (CA) induces whole-body ischemia, which causes damage to multiple organs, including the brain, heart, kidney, and liver [1]. How to reduce damages to these organs in CA is critical to developing better resuscitation strategies to improve patient survival rates, which has not improved over the last few decades [2]. Reducing brain injury is a key issue following cardiopulmonary resuscitation (CPR). After $\mathrm{CPR}$, ischemia-reperfusion injury can cause a series of changes, such as tissue and vascular endothelial cell damage, activation of the coagulation system, and decreased tissue plasminogen activator. Brain damage after CA is typically the result of ischemic or hypoxic injury in vulnerable areas of the brain such as the hippocampus, cortex, and thalamus and triggers a series of pathophysiological processes following CA/CPR. The necrosis and apoptosis of numerous nerve cells lead to a variety of neuronal dysfunctions, including anterograde amnesia, learning difficulties, emotional and social behavioral changes, depression, potentially coma, persistent vegetative state, and death [3]. Therefore, brain damage has been recognized as a major sign of post-CA syndrome, which usually increases mortality in addition to the primary diseases. Effective attenuation of brain injury is one of the key aims of cardiopulmonary-cerebral resuscitation (CPCR).

Adverse events during CA and CPR are characterized by excessive coagulation, inadequate endogenous anticoagulation, and fibrinolysis, as well as an inflammatory syndrome that closely resembles the immunological profile observed in sepsis patients [4]. Nevertheless, anticoagulation or thrombolysis was contraindicated during CPR for many 
years due to the fear of severe bleeding complications. Case reports using thrombolysis showed significantly improved survival for patients after thrombolysis during CPR. Other trials showed that bleeding complications do not occur more frequently after thrombolysis during CPR. Experimental investigations demonstrated that thrombolysis during CPR improves cerebral microcirculation $[5,6]$.

CD40 is a type I phosphoprotein with a molecular weight of $50 \mathrm{kD}$, belonging to the tumor necrosis factor (TNF) receptor superfamily. CD40 is mainly expressed at the surface of B Cells, dendritic cells, and monocytes and is also an important signal recognition receptor for activation and maturation of $\mathrm{T}$ and $\mathrm{B}$ cells $[7,8]$. The dysfunction of the CD40/CD40L system has been reported to participate in the development and progression of atherosclerosis, sepsis, and other inflammatory diseases [8-11]. In the brain, CD40 is mainly expressed in the microglia, neurons, and neuron-like cells [12]. When CD40 binds to CD40L, it may activate a variety of signaling pathways including NF- $\kappa \mathrm{B}$, leading to changes in the expression and function of many procoagulant and proinflammatory genes. So, the costimulatory CD40/CD40L receptor/ligand dyad serves as a well-known link of thrombogenesis and inflammation. Hypercoagulability and inflammation may lead to the production of various inflammatory cytokines and neuronal toxins, further aggravating the inflammatory response in brain tissue after CA. Therefore, in this study, we have conducted experiments on rats to investigate the effect of prophylactic anticoagulation on the brain cells after CPCR and to explore whether it may play a protective role by regulating the level of CD40. Our main goal is to improve post-CA brain injury management.

\section{Materials and Methods}

2.1. Subjects. A total of 70 healthy male Sprague Dawley (SD) rats, aged 8-9 months and weighing 350-450 g, were well fed according to laboratory experiment requirements one week before the experiment, with free access to water in the cages, whereby the temperature was maintained between $15-25$ degrees at a humidity rate of $45-55 \%$.

All animal procedures were approved and conducted in accordance with the Animal Ethics Committee of NingXia Medical School and the National Animals Scientific Procedure Regulations. Animals were provided by the Laboratory Animal Center of NingXia Medical Center.

2.2. Grouping. The SD rats were randomly divided into 4 groups: the normal control group $(n=10$ rats without anesthesia and operation), the sham group ( $n=10$ rats with anesthesia only), the nonanticoagulation group ( $n=25$ rats with anesthesia and CA/CPCR), and the anticoagulation group ( $n=25$ rats with anesthesia and operation with prophylactic heparinization before CA) [13].

During the course of the experiment, there were a total of 9 subjects excluded. There were three cases of liver injury complicated with massive hemorrhage, one case of lung injury, three cases of vascular injury with blood loss of more than $1.5 \mathrm{ml}$, and 2 cases of inadequate ventilation and insufficient oxygen supply. Eventually, there were 10 subjects in the control and sham groups and 25 subjects in the nonanticoagulation and heparin groups. There was no significant difference in the weights among the 4 groups before the start of the experiment.

There was no difference in the weights before and after the experiment in the subjects of the sham group (Table 1). However, asphyxial CA caused a significant decrease in weights in nonanticoagulation and heparin groups after the experiment. But, there was no significant difference between the two groups (Table 1). There were also no significant differences in the baseline MABP, HR, RT, and $\mathrm{P}_{\mathrm{E}} \mathrm{tCO}{ }_{2}$ between nonanticoagulation and heparin groups (Table 2).

2.3. Asphyxial CA Model. In order to setup the respirationCA model, the subjects were stabilized for 5-8 min, and then, the baseline MABP, HR, TR, PEtCO2, and HR were recorded before another intravenous dose of $1 \mathrm{mg} / \mathrm{Kg}$ vecuronuim and after $1 \mathrm{~min}$, mechanical ventilation was stopped, the subject was extubated, and the tracheal catheter was clipped to induce asphyxia. 2-4 min after asphyxia, there would be a brief episode of elevated blood pressure followed by a drop in pressure to null pressure, MABP $\leq 10 \mathrm{mmHgl}$. At the same time, there would have been an episode of progressive tachycardia, which would eventually slow down until CA. The electrocardiogram would show static ECG, ventricular fibrillation, and electrical mechanical dissociation. The apical region heart beats would disappear with cyanosis of the lips and skin mucosa followed by a gradual drop in the rectal temperature.

2.4. CPR. $8 \mathrm{~min}$ after asphyxia, the clipped trachea catheter is opened, and then, 4-5 times of fast ventilation of pure oxygen at a tidal volume of $8-10 \mathrm{ml}$ was performed and, then, maintained at a tidal volume of $1 \mathrm{ml}$ at a mechanical ventilation rate of $60-75$ times $/ \mathrm{min}$. $0.01 \mathrm{~g} / \mathrm{kg}$ adrenaline followed by $1 \mathrm{mmol} / \mathrm{L} . \mathrm{kg} \mathrm{NaHCO}_{3}$ were administered intravenously. At the same time, CPR was performed with cardiac compression with one horizontal finger at the level of the xiphoid process and two fingers on the lateral sides of the sternum at a frequency of 200 beats per minute until independent arterial blood pressure $>60 \mathrm{mmHg}$ and restoration of spontaneous circulation (ROSC). The standards of resuscitation: ECG showed normal QRS waves, palpation of obvious heart throb, cyanosed skin and lips turn rosy, and a mean arterial pressure greater than $60 \mathrm{mmhg}$. Therefore, a controlled asphyxia of 8 min can definitely cause a pulseless period of 4-5 min (CA). After ROSC, high-frequency ventilation of $60-80$ times/min is maintained for $10 \mathrm{~min}$, and then, the ventilation frequency is adjusted to maintain a PEtCO2 of $30-35 \mathrm{mmHg}$.

2.5. Postresuscitation Management. Mechanical ventilation is maintained for $60 \mathrm{~min}$ after ROSC, and there is no need to use muscle relaxants after spontaneous respiration returns. There is no requirement to use vasosuppressors when MABP 
TABLE 1: Comparison of the baseline parameters and weights among different groups (mean \pm SD).

\begin{tabular}{lccc}
\hline & Sham $(n=10)$ & Nonanticoagulation $(n=25)$ & Heparin $(n=25)$ \\
\hline Mean arterial blood pressure $(\mathrm{MABP}, \mathrm{mmHg})$ & $90 \pm 6$ & $89 \pm 7$ & $88 \pm 8$ \\
Heart rate (HR, beats/min) & $321 \pm 21$ & $319 \pm 26$ & $322 \pm 24$ \\
End-tidal carbon dioxide $\left(\mathrm{P}_{\mathrm{E}} \mathrm{tCO}, \mathrm{mmHg}\right)$ & $36 \pm 4$ & $36 \pm 5$ & $35 \pm 5$ \\
Fraction of inspired oxygen $(\mathrm{FiO}, \%)$ & $79 \pm 3$ & $80 \pm 3$ & $80 \pm 3$ \\
Rectal temperature (RT) & $37.9 \pm 0.4$ & $38.1 \pm 0.6$ & $37.9 \pm 0.5$ \\
Baseline weight $(\mathrm{g})$ & $390 \pm 28$ & $387 \pm 29$ & $394 \pm 27$ \\
Weight after 72 $\mathrm{h}$ & $391 \pm 26$ & $343 \pm 22^{*}$ & $344 \pm 19^{*}$ \\
\hline
\end{tabular}

Note: after $72 \mathrm{~h}$, there was significant difference in the weight of in the nonanticoagulation and heparin groups compared with the weight before the experiment, $P<0.05$.

TABLE 2: Changes in the vitals and other parameters during CPR in nonanticoagulation and heparin groups (mean \pm SD).

\begin{tabular}{|c|c|c|c|c|c|c|c|}
\hline & & $\begin{array}{c}\text { Before } \\
\text { asphyxiation }\end{array}$ & ROSC & $\begin{array}{l}10 \text { min after } \\
\text { ROSC }\end{array}$ & $\begin{array}{l}30 \text { min after } \\
\text { ROSC }\end{array}$ & $\begin{array}{l}60 \text { min after } \\
\text { ROSC }\end{array}$ & $\begin{array}{c}\text { After } \\
\text { ventilation }\end{array}$ \\
\hline \multirow{4}{*}{$\begin{array}{l}\text { III } \\
(n=10)\end{array}$} & MABP (mmHg) & $89 \pm 7$ & $125 \pm 13$ & $96 \pm 4$ & $87 \pm 6$ & $77 \pm 7$ & $77 \pm 8$ \\
\hline & HR (bpm) & $325 \pm 23$ & $419 \pm 45$ & $349 \pm 10$ & $322 \pm 24$ & $314 \pm 27$ & $286 \pm 18$ \\
\hline & $\begin{array}{l}\mathrm{PEtCO}_{2} \\
(\mathrm{mmHg})\end{array}$ & $36 \pm 5$ & $44 \pm 4$ & $37 \pm 4$ & $33 \pm 2$ & $36 \pm 3$ & $35 \pm 3$ \\
\hline & $\mathrm{RT}\left({ }^{\circ} \mathrm{C}\right)$ & $38.1 \pm 0.6$ & $37.5 \pm 0.4$ & $37.7 \pm 0.4$ & $37.9 \pm 0.2$ & $38.2 \pm 0.4$ & $38.4 \pm 0.3$ \\
\hline \multirow{4}{*}{$\begin{array}{l}\text { IV } \\
(n=21)\end{array}$} & MABP $(\mathrm{mmHg})$ & $88 \pm 8$ & $145 \pm 10^{* *}$ & $98 \pm 5$ & $99 \pm 7$ & $86 \pm 6$ & $79 \pm 9$ \\
\hline & HR (bpm) & $322 \pm 19$ & $409 \pm 39$ & $358 \pm 11$ & $318 \pm 21$ & $317 \pm 25$ & $289 \pm 17$ \\
\hline & $\begin{array}{l}\mathrm{PEtCO}_{2} \\
(\mathrm{mmHg})\end{array}$ & $35 \pm 5$ & $41 \pm 5$ & $37 \pm 4$ & $34 \pm 2$ & $35 \pm 2$ & $35 \pm 3$ \\
\hline & $\mathrm{RT}\left({ }^{\circ} \mathrm{C}\right)$ & $37.9 \pm 0.5$ & $37.7 \pm 0.6$ & $37.8 \pm 02$ & $37.9 \pm 0.1$ & $38.4 \pm 0.5$ & $38.5 \pm 0.4$ \\
\hline
\end{tabular}

Note: there was a significant difference in MABP between the two groups immediately after ROSC, ${ }^{* *} P<0.01$, no significant difference among the other parameters.

$<60 \mathrm{mmHg}$, and slow intravenous administration of isotonic saline at $2-3 \mathrm{ml} / \mathrm{kg}$ would maintain normal MABP. $60 \mathrm{~min}$ after stopping the mechanical ventilation, the trocars are removed and the blood vessels are clamped and the skin is sutured. At this time, $75-80 \%$ oxygen is supplied using the " $T$ "-shape tube. When the breathing rate is greater than 60 beats/min and less than 120 beats/min, the pharyngeal and corneal reflexes would recover, and there would not be any bradycardia ( $\mathrm{HR}<300$ beats/min). After breathing atmospheric air without abnormal findings for $5 \mathrm{~min}$, extubation is performed.

After extubation, the rats are kept in the prone position into a box with $50 \%$ oxygen for $30 \mathrm{~min}$ and, then, returned to the dark and quiet cages with air to breathe and 12-hour day and night alternation. During 60-120 min after ROSC, an isotonic saline solution is administered hypodermically at a dose of $20 \mathrm{ml} / \mathrm{kg}$ to prevent dehydration, and this procedure is repeated once a day, until the rats can feed on water.

In case of any of the following events, the subject would be excluded from the study: bleeding of more than $1.5 \mathrm{ml}$ due to the operation; injury other than the surgery (respiratory tract, lung, and liver damage); and inadequate ventilation or limited oxygen supply. If the overall CPR time exceeds more than $2 \mathrm{~min}$, resuscitation is considered to be failed. The subjects surviving for less than $72 \mathrm{~h}$ after CPR have to be excluded from the neurological deficit (ND) and histopathological damage (HD) score assessment since there would be no substantial pathological damage and behavioral problems. The ND score of the 4 groups before and $72 \mathrm{~h}$ after the experiment was assessed according to the rats asphyxia model ND scoring criteria by Laurence Katz et al. After assessing the $72 \mathrm{~h}$ ND score, the 4 groups of rats were anesthetized by an intraperitoneal injection of sodium $45 \mathrm{mg} / \mathrm{Kg}$ followed by intubation and mechanical ventilation. A left sternal thoracotomy was performed and using a $20 \mathrm{D}$ trocar, and $5 \mathrm{ml}$ of $10 \%$ paraformaldehyde $+1 \mathrm{ml}$ of $25 \%$ glutaraldehyde $+25 \mathrm{ml}$ of $0.2 \mathrm{M}$ phosphate buffer $+19 \mathrm{ml}$ of double distilled water were infused in the aorta, allowing for $50 \mathrm{ml}$ of cerebral perfusion. Then, the brain tissues were harvested and placed in 3\% paraformaldehyde (PH 7.4, 4 degrees Celcius) for 24 hours and, then, embedded in paraffin. According to the anatomical Atlas of the rat brain by Paxinos [14], the coronal presentation was prepared using $6 \mu \mathrm{m}$ paraffin sections across the 19, 29, 36, 42 , and 63 regions, and then, Nissl's staining was performed. The extent of histopathological damage of the hippocampus (CA1-3) was, then, observed under microscopy. Eventually, using the Laurence Katz HD scoring system and standards, the histological damage in the hippocampus, cortex, thalamus, shell caudate nucleus, and cerebellum was assessed and scored. After cerebral perfusion, two rats were taken from each group, and the hippocampus (CA1-3) was retrieved from the same area and prepared into $1 \mathrm{~mm}^{3}$ electron microscopy slides.

2.6. Light Microscope Sample Preparation. The brain tissue was fixed in $10 \%$ neutral formalin $(24 \mathrm{~h})$. After fixation, the 
TABle 3: The comparison of the CPR success and $72 \mathrm{~h}$ survival rates of nonanticoagulation and heparin groups.

\begin{tabular}{|c|c|c|c|c|c|}
\hline & $\begin{array}{l}\text { Time from asphyxiation to } \\
\text { CA (s) }\end{array}$ & $\begin{array}{c}\text { Time from CPR to } \\
\text { ROSC (s) }\end{array}$ & $\begin{array}{l}\text { Time of tracheal } \\
\text { extubation }(\mathrm{h})\end{array}$ & $\begin{array}{c}\text { CPR success rate } \\
(\%)\end{array}$ & $\begin{array}{c}72 \mathrm{~h} \text { survival rate } \\
(\%)\end{array}$ \\
\hline Nonanticoagulation & $167 \pm 20^{* *}$ & $81.3 \pm 20.4^{* *}$ & $6.9 \pm 1.4^{* *}$ & $64(16 / 25)^{*}$ & $62.6(10 / 16)^{*}$ \\
\hline Heparin & $196 \pm 32$ & $42.7 \pm 15.8$ & $4.8 \pm 1.2$ & $92(23 / 25)$ & $91.3(21 / 23)$ \\
\hline
\end{tabular}

Note: the measurement data are expressed as mean $\pm \mathrm{SD}$, while the count data is expressed as percentages. There is significant difference in the time from asphyxiation to CA and from CPR to ROSC between groups of nonanticoagulation and heparin, ${ }^{* *} P<0.01$; the CPR success rate and survival rate 72 hours after ROSC between the two groups were also significantly different, $P<0.05$.

TABLE 4: The ND scores of groups III and IV at different time points after CPR (mean \pm SD\%).

\begin{tabular}{|c|c|c|c|c|c|}
\hline Groups & Before anesthesia & $2 \mathrm{~h}$ after ROSC & $24 \mathrm{~h}$ after ROSC & $48 \mathrm{~h}$ after ROSC & $72 \mathrm{~h}$ after ROSC \\
\hline Control & $0 \pm 0$ & - & - & - & $0 \pm 0$ \\
\hline Sham & $0 \pm 0$ & - & - & - & $0 \pm 0$ \\
\hline Nonanticoagulation & $0 \pm 0$ & $62.9 \pm 8.2$ & $39.2 \pm 8.3$ & $28.2 \pm 5.3$ & $24 \pm 7.1$ \\
\hline Heparin & $0 \pm 0$ & $49.1 \pm 9.7^{* *}$ & $18.1 \pm 6.7^{* *}$ & $4.9 \pm 3.9^{* *}$ & $4.5 \pm 2.1^{* *}$ \\
\hline
\end{tabular}

Note: before anesthesia, the ND score of all the groups was $0+0 \%$; there was a significant difference in ND scores between groups of nonanticoagulation and heparin at $2 \mathrm{~h}, 24 \mathrm{~h}, 48 \mathrm{~h}$, and $72 \mathrm{~h}$ after $\mathrm{CPR},{ }^{* *} \mathrm{P}<0.0,1$.

TABLE 5: Ischemic neuron cell count from different brain regions at different time points after ROSC in the 4 groups (mean $t$ SD\%).

\begin{tabular}{lccccc}
\hline Group & Hippocampus & Cortex & Thalamus & Cerebellum & Shell caudate nucleus \\
\hline Control & $0 \pm 0$ & $0 \pm 0$ & $0 \pm 0$ & $0 \pm 0$ & $0 \pm 0$ \\
Sham & $0 \pm 0$ & $0 \pm 0$ & $0 \pm 0$ & $0 \pm 0$ & $0 \pm 0$ \\
Nonanticoagulation & $65.7 \pm 13.46^{* *}$ & $28.3 \pm 7.04^{* *}$ & $30 \pm 5.19^{* *}$ & $18.7 \pm 3.33^{* *}$ & $18.4 \pm 5.62^{* *}$ \\
Heparin & $26.95 \pm 8.42$ & $17.53 \pm 6.43$ & $23 \pm 4.47$ & $12.74 \pm 2.84$ & $12.95 \pm 3.73$ \\
\hline
\end{tabular}

Note: upon comparing the HD count from the different brain regions, there is a significant difference between the groups of nonanticoagulation and heparin, ${ }^{* *} P<0.01$.

brain tissue was passed through an ethanol gradient for dehydration and, then, soaked in xylene and embedded in paraffin followed by slicing. Then, the sample was dewaxed in xylene and rehydrated through decreasing concentrations of ethanol and stained with HE.

After rehydration with ethanol, the samples were sectioned, and then, immunohistochemical staining was performed and the slice was mounted. The rate of immunohistochemical expression of TNF- $\alpha$, IL-1 in the neurons of the hippocampus (CA1-3) was statistically analyzed to assess the extent of brain histological damage (the TNF- $\alpha$, IL- $1 \beta$ reagents were provided by the Wuhan Ph.D. Biological Engineering Co., Ltd.).

2.7. Sample Preparation for Electron Microscopy. $1 \mathrm{~mm}^{3}$ of hippocampus of the same area was retrieved, and the cortex was first fixed in $2.5 \%$ glutaraldehyde followed by $1 \%$ osmium tetroxide. The sample was dehydrated through grade concentration of ethanol and soaked in epoxy resin and embedded and, then, sliced using the LKB ultrathin slicer followed by lead staining. The H-600-4 transmission electron microscope was used to observe the ultrastructural changes in the hippocampal cells.

2.8. The Brain Histopathological Damage (HD) Assessment. Optical microscopy and electron microscopy were performed to assess the HD scores according to the standards by
Laurence Katz. Using the three-grid method, all the neurons (normal and ischemic) of all sides of the respective brain region were counted (in a range of $0.25 \times 0.05 \mathrm{~mm}$ at a magnification $\times 200$ ). The proportion of ischemic neurons in each brain region was represented as a percentage of the total neuron count of the region. The average HD score of the ischemic neurons from the five different cerebral regions in each brain tissue section was obtained. The rate of immunohistochemical expression of TNF- $\alpha$, IL- $1 \beta$ in the neurons from the hippocampus (CA1-3) was statistically analyzed. Under three random view slides using the three-grid counting method, all positive and negative staining neurons (normal and ischemia) from each side of the corresponding brain regions were counted $(0.25 \times 0.05 \mathrm{~mm}$ range at $\times 200$ magnification), hence determining the percentage of positive staining cells. The number of cases in nonanticoagulation and heparin groups still surviving $72 \mathrm{~h}$ after successful CPR was recorded.

2.9. Western Blotting. An equal amount $(60 \mu \mathrm{g})$ of the total protein was separated by SDS-polyacrylamide gel electrophoresis (SDS-PAGE) (Beyotime Biotech, China) and then transferred onto a polyvinylidene difluoride (PVDF) (Pell, USA) membrane after electrophoresis. The membranes were immunoblotted with Abs against CD40, p-p65, and GAPDH (human monoclonal antibody, Sigma, USA) followed by a horseradish peroxidase-conjugated secondary. The 


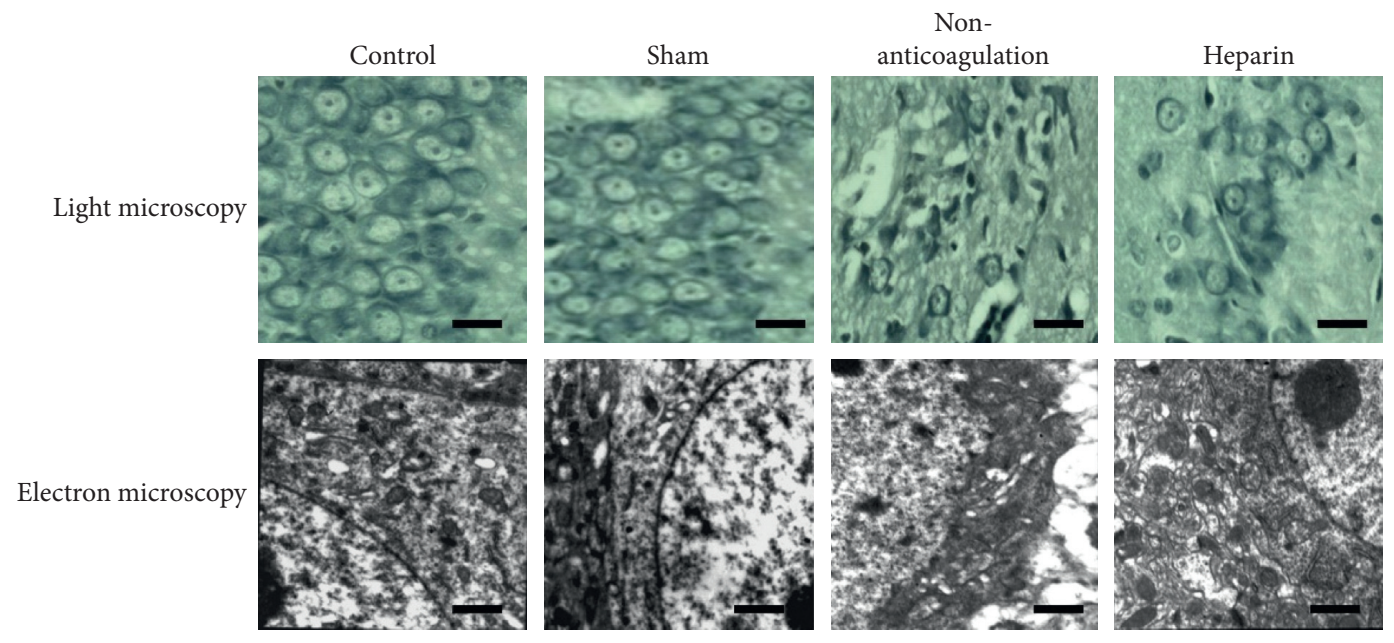

Figure 1: Heparin alleviated the brain damage induced by asphyxial CA. Hippocampus (CA1-3) of SD rats was observed by using the light microscope and electron microscopy from control, sham, noncoagulation, and heparin groups. Scale bar: $50 \mu \mathrm{m}$.

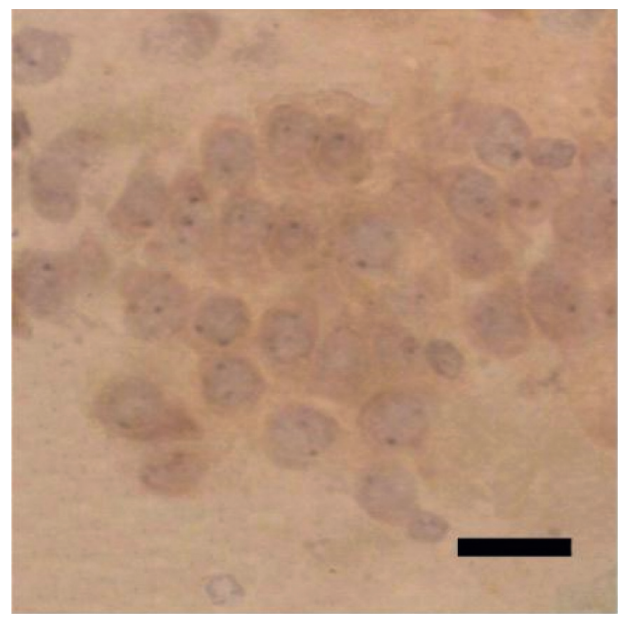

(a)

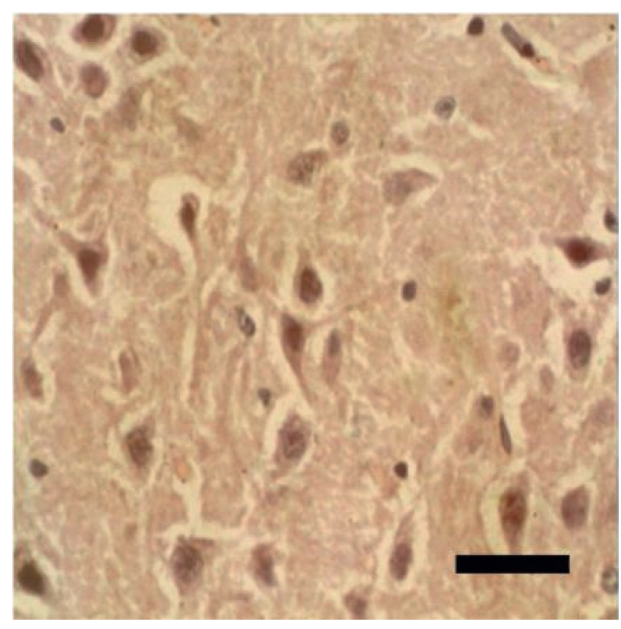

(c)

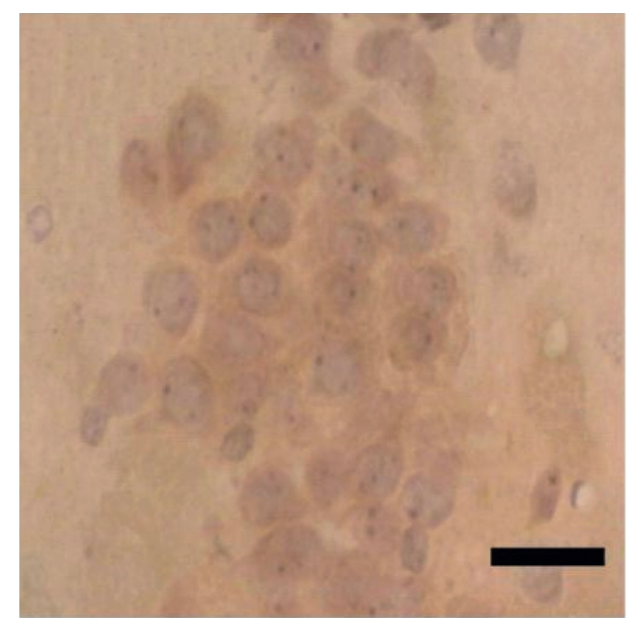

(b)

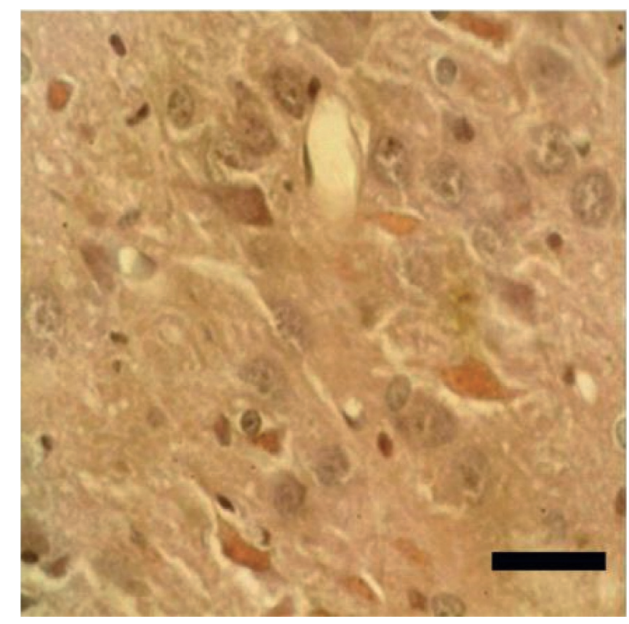

(d)

FIGURE 2: Heparin treatment reduced the expression of TNF- $\alpha$ in the hippocampus. Immunohistochemical staining of hippocampus (CA1-3) from control, sham, noncoagulation, and heparin groups is shown. Scale bar: $50 \mu \mathrm{m}$. (a) Control, (b) sham, (c) noncoagulation, and (d) heparin. 


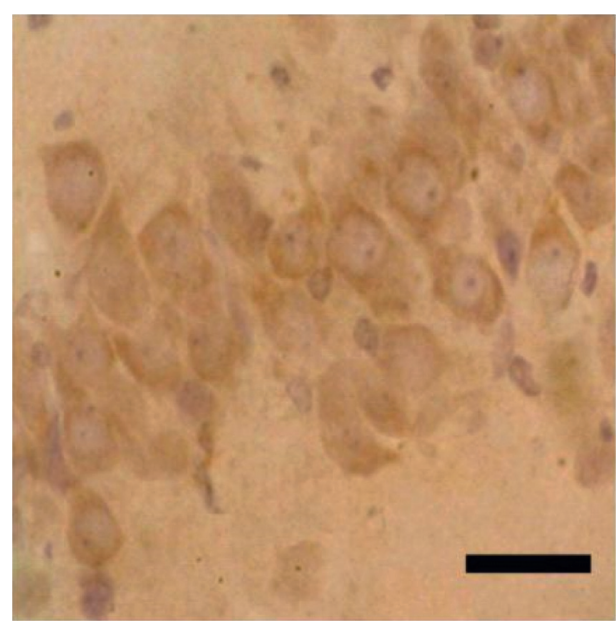

(a)

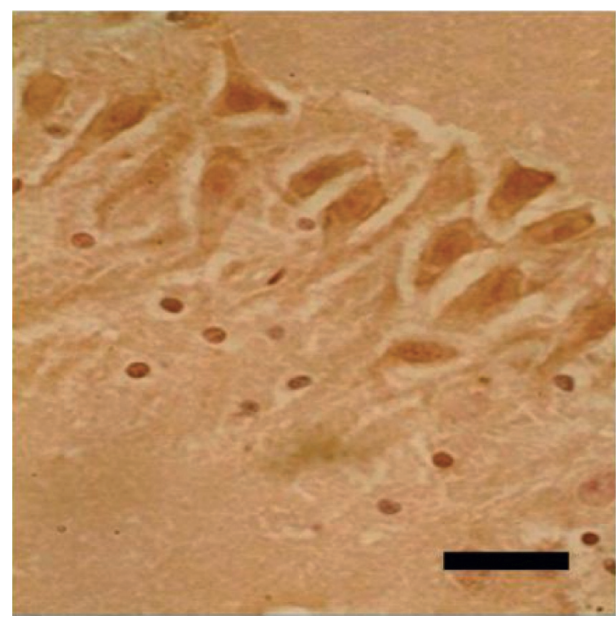

(c)

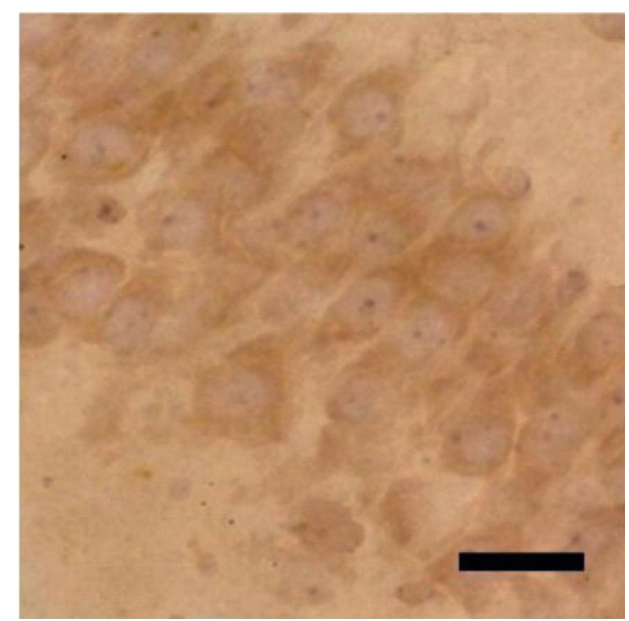

(b)

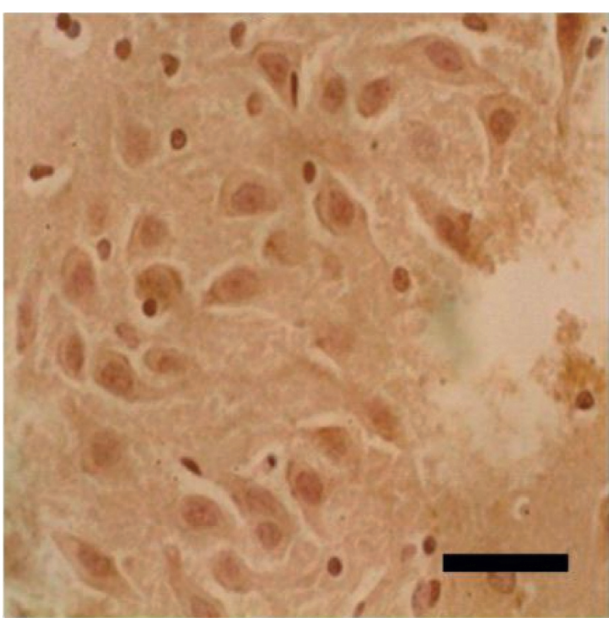

(d)

FIGURE 3: Heparin treatment reduced the expression of IL-1 $\beta$ in the hippocampus. Immunohistochemical staining of hippocampus (CA1-3) from control, sham, noncoagulation, and heparin groups is shown. Scale bar: $50 \mu \mathrm{m}$. (a) Control, (b) sham, (c) noncoagulation, and (d) heparin.

immunoblots were detected by using a Bio-Rad Calibrated Densitometer.

2.10. Real-Time PCR Analysis. Real-time PCR was performed according to the manufacturer's instructions using the ABI 7300 real-time PCR system with the SYBR Green method. Relative abundances of IL- $1 \beta$, IL-6, and VCAM-1 mRNAs from brain tissues were normalized to the expression level of GAPDH. All amplification reactions were performed in triplicate.

2.11. Statistical Analysis. SPSS11.5 was used for the statistical analysis. The data were expressed as the mean \pm SD. The differences among the groups were compared using a one-way ANOVA. Unpaired Student' $t$-test was used for direct comparisons. The Kruskal-Wallis analysis was used to compare the differences in the ND and HD scores from different groups. Fisher's exact test was used to compare the success rate of CPR and the $72 \mathrm{~h}$ survival rate differences in groups of nonanticoagulation and heparin. Spearman's rank correlation coefficient was used to analyze the correlation between the ND and HD scores of groups of nonanticoagulation and heparin. $P<0.05$ was considered to be statistically significant.

\section{Results}

3.1. Heparin Reduced the Recovery Time after Standard CPR. There was no significant difference between the baseline physiological indexes of nonanticoagulation and heparin groups before the start of asphyxiation (Table 2). After asphyxiation, CA (MABP $\leq 10 \mathrm{mmHg})$ happened after $167 \pm 20 \mathrm{~s}$ and $196 \pm 32 \mathrm{~s}$ in the nonanticoagulation group and heparin group, respectively, manifesting as electrical mechanical dissociation or CA. After standard CPR, it was after $81.3 \pm 15.8$ and $42.7 \pm 20.4 \mathrm{~s}$ that the asphyxia-induced CA was reversed, and heparin significantly reduced the recovery time, $P<0.01$ (Table 3 ). After CPR, out of the 25 subjects in the nonanticoagulation group, only 16 animals recovered spontaneous respiration successfully, and 9 subjects failed to recover. After CPR, out of the 25 subjects of the heparin group, 23 subjects recovered successfully, while 2 


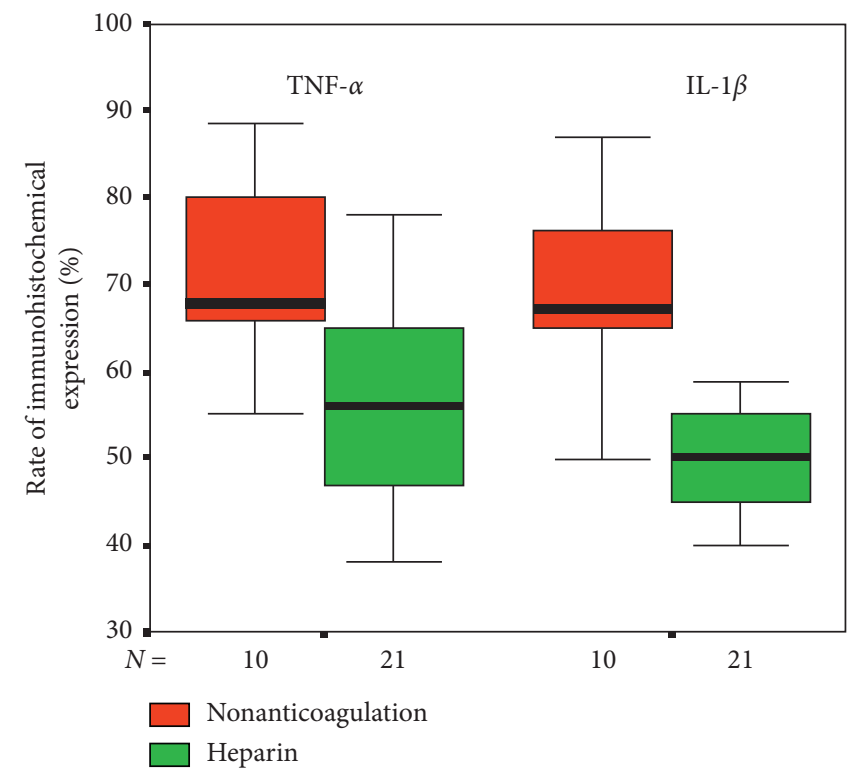

Figure 4: Heparin treatment decreased the percentage of positively stained TNF- $\alpha$ and IL- $1 \beta$ particles in the hippocampus. The box-plot shows the positive rates of TNF- $\alpha$ and IL- $1 \beta$ in noncoagulation and heparin groups.

subjects failed. $72 \mathrm{~h}$ after ROSC, only 10 out of the 16 subjects from the nonanticoagulation group survived while 6 died out cardiac failure or arrhythmia, whereby in the heparin group, 21 out of the 23 subjects survived while 2 died of heart failure (heart failure or arrhythmia occurred in between 10 and $50 \mathrm{~min}$ after ROSC). Therefore, there were, respectively, 10 and 21 subjects from the nonanticoagulation group and heparin group that completed the ND and HD score assessment.

3.2. Heparin Alleviated the Brain Damage Induced by Asphyxial CA. There were no anesthesia and vascular catheter used in the control group. Hence, except from weight, ND, and HD scores, there were no other experimental parameters to be observed. The different parameters in the sham group were all stable within the 60 min on life support, with complete recovery of consciousness without any nervous system dysfunction or damage $0.5-1 \mathrm{~h}$ after extubation. At the end of the experiment, the ND score for the control group and sham group were 0 , respectively, and the overall $\mathrm{HD}$ score from the 5 brain regions was also 0 (Tables 4 and 5).

Immediately after ROSC, there was a significant difference in the MABP from the nonanticoagulation group and heparin group, while there was no difference in the other parameters at other time points (Table 2). The time intervals from asphyxiation to CA, from CPR to ROSC, and from mechanical ventilation to extubation were significant different between the nonanticoagulation group and heparin group (Table 3 ). The heparin group also showed a better CPR success rate of CPR and $72 \mathrm{~h}$ survival rate than those in the nonanticoagulation group (Table 3 ). There was no difference in the ND score among the 4 groups before the experiment and between the control group and sham group at the end of the experiment (Table 4). There was a significant difference in the ND scores $2 \mathrm{~h}, 24 \mathrm{~h}, 48 \mathrm{~h}$, and $72 \mathrm{~h}$ after ROSC between the nonanticoagulation group and heparin group (Table 4). At the end of the experiment, the HD score for the hippocampus (CA1-3), cortex, thalamic reticular nucleus, lateral shell of caudate nucleus, and cerebella was 0 , showing no significant difference among all groups, while there was significant difference between the nonanticoagulation group and heparin group (Table 5). After CA, the nervous system damages in the nonanticoagulation group and heparin group were identified by unilateral or bilateral foot spastic paralysis, but there was no such manifestations in the subjects from the sham group, suggesting that the damage to the nervous system in the nonanticoagulation group and heparin group was, indeed, caused by CA which led to brain damage and not due to the operation. There was a significant correlation between the ND and HD scores of the 2 groups: nonanticoagulation group $r=0.86, P=0.0018$; heparin group $r=0.81, P=0.0027$.

After the experiment, the structure of the nerve cells from the hippocampus (CA1-3) collected from the control and sham groups was normal under light and electron microscopy (Figure 1). In the nonanticoagulation group and heparin group, there were different degrees of damage seen in the hippocampus neurons (CA1-3) under a light microscope and electron microscope, the extent of damage being more serious in the nonanticoagulation group (Figure 1).

After immunohistochemical staining, there was no positive expression of TNF- $\alpha$ (Figure 2 ) and IL-1 $\beta$ (Figure 3 ) in the hippocampus (CA1-3) of the control and sham groups. However, there was a different extent of positive expression of TNF- $\alpha$ (Figure 2 ) and IL-1 $\beta$ (Figure 3 ) in the hippocampus (CA1-3) of the nonanticoagulation group and heparin group, whereby the rate of positive expression was significantly higher in the nonanticoagulation group. The percentage of positively stained TNF- $\alpha$ and IL- $1 \beta$ particles in 

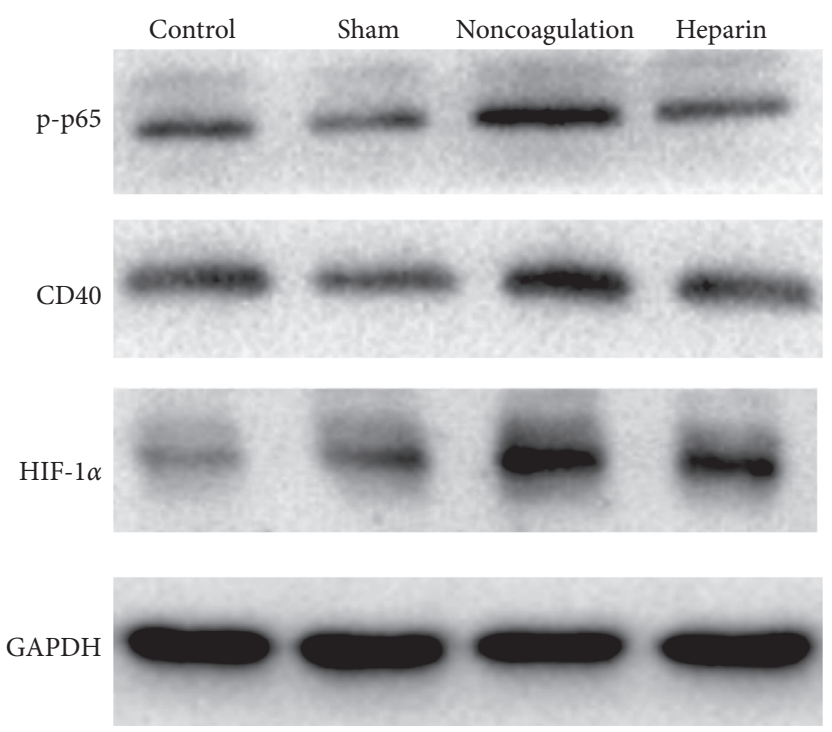

(a)

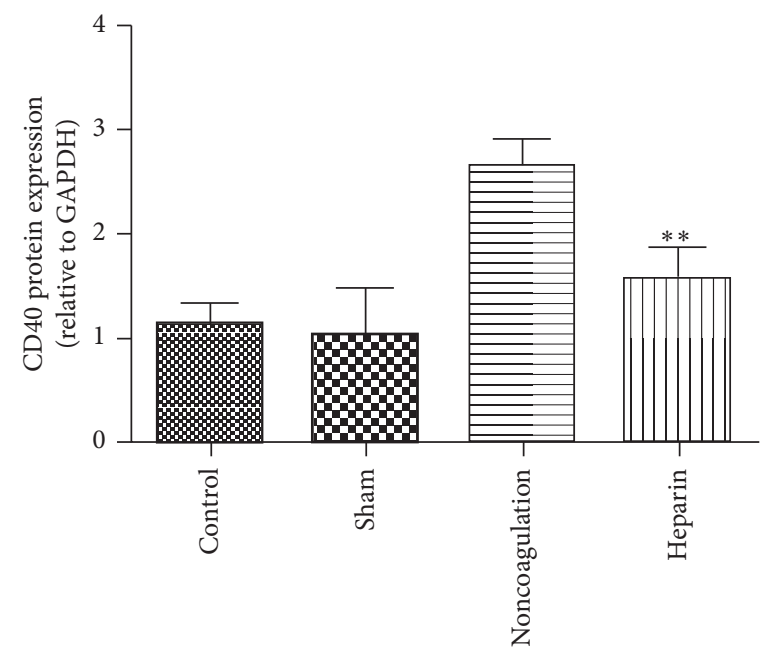

(c)

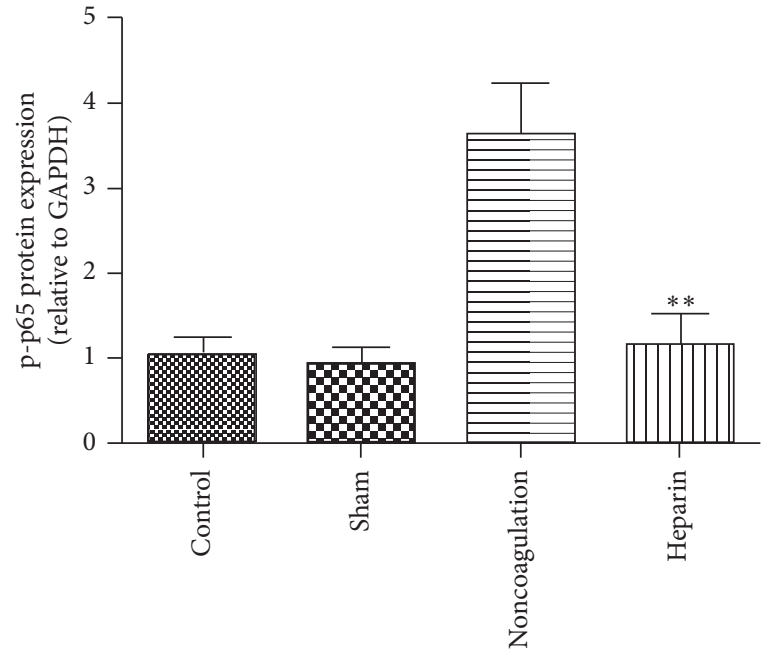

(b)

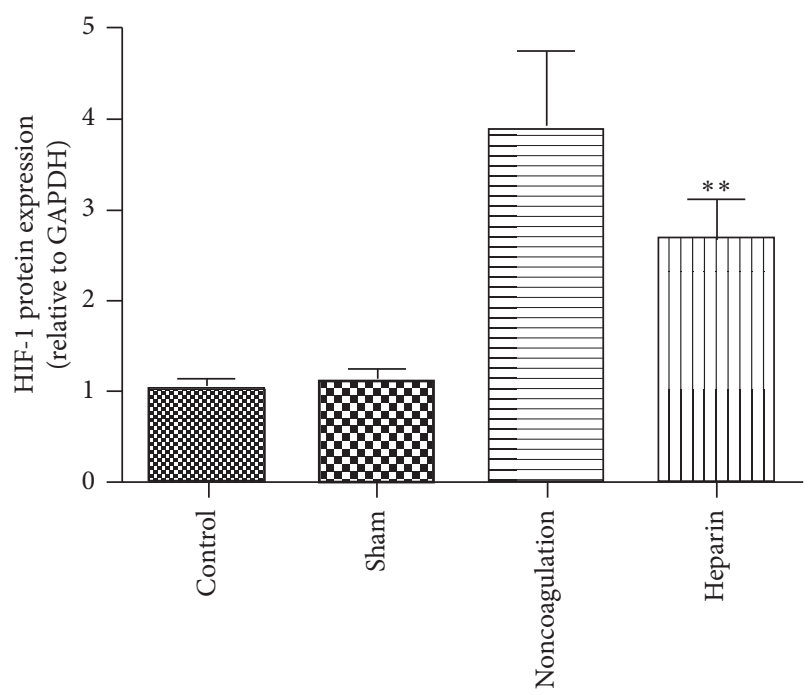

(d)

FIgURE 5: Heparin treatment inhibited the expressions of CD40, NF- $\kappa$ B, and HIF- $1 \alpha$ induced by asphyxial CA. (a) Representative immunoblots of CD40, p-p65, and HIF- $1 \alpha$ in the hippocampus. Statistical analysis of CD40 (b), p-p65 (c), and HIF-1 $\alpha$ (d) expressions. Data are expressed as mean $\pm \mathrm{SD}, n=4 .{ }^{* *} P<0.01$ vs. nonanticoagulation.

the nonanticoagulation group and heparin group was also significantly different, and heparin treatment significantly decreased the percentage of positively stained cells (Figure 4).

\subsection{The Protective Effects of Heparin Are Partially Dependent} on the Suppression of CD40 and HIF-1 $\alpha$. We, then, tried to find the related signal pathway that was involved in the enhanced inflammatory cytokines. It is well established that the activation of the CD40/CD40L pathway is associated with elevated inflammatory cytokines in a wide range of diseases, such as sepsis, ischemic stroke, and hypertension. Ischemia and hypoxia can also induce the expression of HIF$1 \alpha$, causing major brain damage after CA. So, we collected the brain tissues and detected the expressions of CD40, NF- $\kappa \mathrm{B}$, and HIF- $1 \alpha$. As shown in Figures $5(\mathrm{a})-5(\mathrm{~d})$, the expressions of CD40, NF- $\kappa$ B p-p65, and HIF- $1 \alpha$ were comparable in the brain tissues form control and sham groups. However, asphyxial CA caused a significant increase in CD 40 , NF- $\kappa$ B p-p65, and HIF- $1 \alpha$ expressions in brain tissues from the nonanticoagulation group. Treatment of the subject mice with heparin could partially reverse the increased expressions of CD40, NF- $\kappa \mathrm{B}$ p-p65, and HIF- $1 \alpha$, indicating the inhibitory effects of heparin on CD40/NF- $\kappa$ B and HIF$1 \alpha$ pathways. We also used reverse transcriptase PCR to detect some downstream genes of the NF- $\kappa \mathrm{B}$ pathway, including IL-1 $\beta$, IL-6, and VCAM-1, in order to show that the NF- $\kappa \mathrm{B}$ expression is functionally altered. As expected, their expressions were significantly elevated after asphyxial CA, and heparin significantly inhibited the upregulation of IL-1 $\beta$, IL- 6 , and VCAM- 1 . 


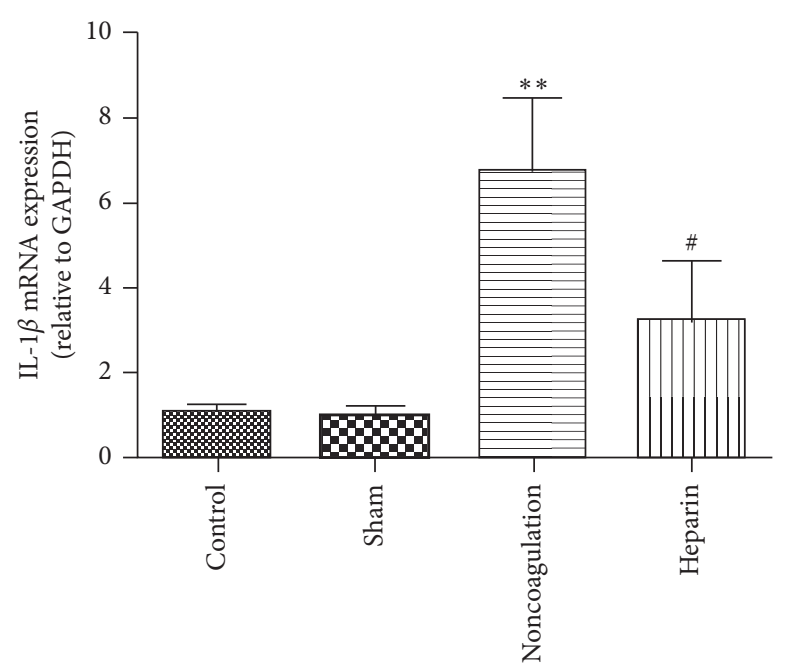

(a)

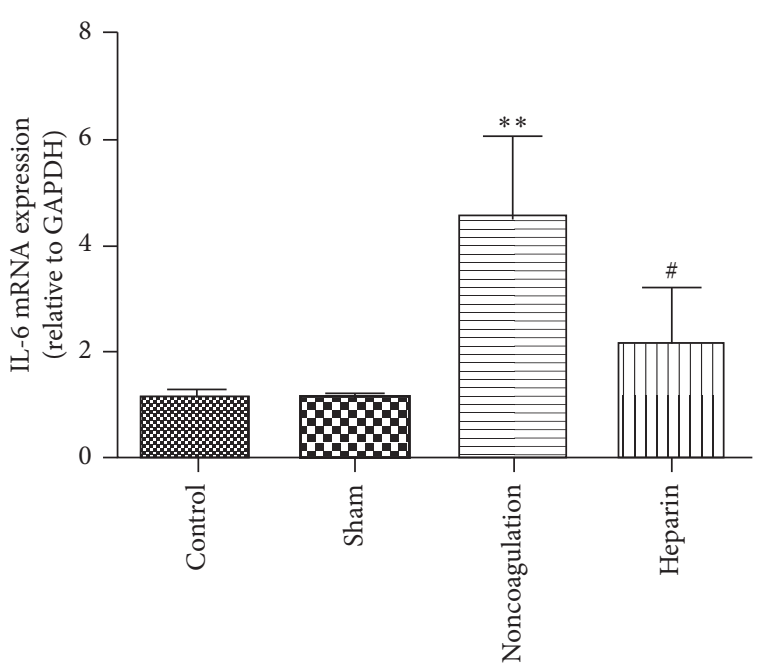

(b)

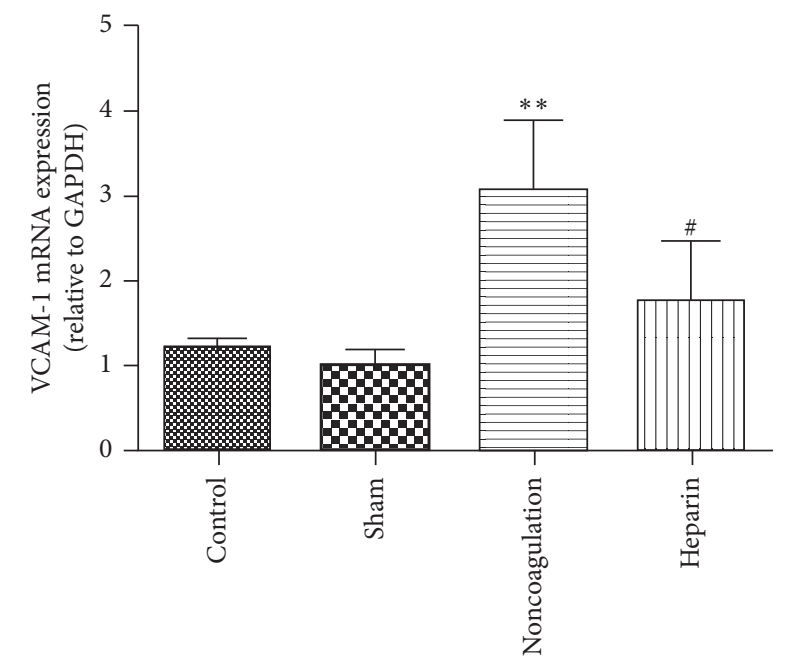

(c)

FIgURE 6: Heparin treatment inhibited the mRNA expressions of IL-1 $\beta$, IL-6, and VCAM-1induced by asphyxial CA. RNA from brain tissues of different groups was analyzed by real-time PCR for IL-1 $\beta$ (a), IL-6 (b), and VCAM-1 (c). Data are expressed as mean \pm SD, $n=4$. ${ }^{* *} P<0.01$ vs. nonanticoagulation.

\section{Discussion}

In this study, we mainly focused on the effects of anticoagulant therapy on the brain function recovery after CPCR and its association with CD40. Based on previous studies, $50-60 \%$ of in-hospital deaths after CPR are related to nerve injury, while some are due to irreversible brain damage and residual serious sequelae [15]. Therefore, in a quest to attain maximum possible recovery for the patients, including functional and social recovery has been the focus of attention in the field of medicine and research [16-21]. Even though they can alter cerebral resuscitation prognosis up to some extent, they still cannot manage the microcirculation dysfunction after CA and CPCR. Due to the activation of the coagulation system and inhibition of endogenous fibrinolysis, $5-10 \mathrm{~min}$ after CA, there is cerebral microcirculation disturbance and microthrombosis. Therefore, thrombolytic therapy may be a significant improvement in the outcome of cerebral resuscitation, but there is a lack of randomized controlled experiments to support [22]. In line with this hypothesis, our data shows that anticoagulation significantly promoted the recovery of myocardial contractile function, reduced brain tissue damage after $\mathrm{CPR}$, and may eventually improve the prognosis of CPR.

After determining the therapeutic effect of anticoagulation on asphyxial CA-induced brain damage, we tried to find the underlying molecular mechanism by which heparin exerts its protective effects. CD40 protein is a type I transmembrane receptor, belonging to the TNF receptor superfamily. CD40L is an endogenous ligand of CD40, belonging to type II transmembrane protein, TNF superfamily [23]. Indeed, large amounts of research have revealed the substantial role of CD40 in the process of sepsis, atherosclerosis, ischemic stroke, and Alzheimer's disease $[9,11,24,25]$. Above all, the CD40/CD40L system serves as a vital link between inflammation and thrombogenesis. So, in this study, we collected the brain tissues from all the 4 groups to detect the CD40 protein levels and its downstream NF- $\kappa \mathrm{B}$ 
signaling. As expected, asphyxial CA caused a significant increase in the CD40 expression accompanied by a significant activation of the NF- $\kappa$ B pathway and anticoagulation with heparin before CA could partially reverse the increased CD40 expression and inhibited the activation of NF- $\kappa \mathrm{B}$ pathway, suggesting that the effect of heparin to reverse asphyxial CA-induced brain damage is partly dependent on CD40 inhibition.

IL- $1 \beta$ and TNF- $\alpha$ are the major inflammatory cytokines released by immune cells and local cells in many inflammatory diseases following the activation of CD40 signaling. Our observation indicated that IL- $1 \beta$ and TNF$\alpha$ positive staining rates were decreased in brain tissues from the anticoagulation group than that from the nonanticoagulation group, further supporting the involvement of CD40 in heparin-mediated protective effects. The anticoagulation group also showed less damage of neuron compared with the nonanticoagulation group, suggesting that anticoagulation may reduce the expressions of IL- $1 \beta$ and TNF- $\alpha$, thus reducing brain damage caused by CA. In our study, we also observed that heparin caused significant changes in HIF- $1 \alpha$ protein, suggesting that anticoagulant therapy may affect the prognosis of CPR through more complex molecular mechanisms. However, the underlying mechanism is still unclear and needs further research.

Although some meaningful data have been obtained, there are still some limitations in our study. For example, different types of cells in the hippocampus have different tolerance to CA-induced ischemia and hypoxia. Future research will, therefore, focus on understanding the exact molecular mechanisms by which anticoagulants such as heparin combat brain damage caused by CA.

Moreover, complementary and alternative medicine (CAM) is more and more widely used in the treatment of chronic and disabling diseases and is also used for acute diseases during convalescence [14, 26, 27]. CAM tends to be less toxic and less invasive, and CAM is conducive to solving the side effects, environmental pollution, and economic problems of drugs. It has been confirmed by some studies that the combination of Western medicine and CAM therapy is beneficial to the recovery of cerebral and neurological functions in stroke patients $[28,29]$. Therefore, future studies should investigate whether heparin combined with CAM can further improve ischemic and anoxic injury of brain cells after CPR.

\section{Conclusions}

In summary, anticoagulation can improve the outcome and prognosis of brain ischemia, including the improvement of neurological function recovery and prevention of morphological and immunohistochemical injury on the brain, while significantly increasing the success rate of CPR. Heparin anticoagulant therapy may improve the prognosis of asphyxial CA by inhibiting IL- $1 \beta$, CD 40 , NF- B, HIF- 1 , and other signaling pathways. Our results suggest heparin anticoagulation might be an important supplement to the treatment strategy for the cerebral recovery after CPR.

\author{
Abbreviations \\ CA: Cardiac arrest \\ CPR: Cardiopulmonary resuscitation \\ CPCR: Cardiopulmonary-cerebral resuscitation \\ $\mathrm{PEtCO}_{2}$ : End-tidal carbon dioxide partial pressure \\ TR: Rectal temperature \\ MABP: Mean arterial blood pressure \\ HR: Heart rate \\ RR: $\quad$ Respiration rate \\ ECG: Electrocardiogram \\ EOSC: Restoration of spontaneous circulation \\ HD: Histopathological damage \\ ND: Neurological deficit.
}

\section{Data Availability}

Data can be obtained from the corresponding author upon reasonable request.

\section{Conflicts of Interest}

The authors declare that they have no conflicts of interest.

\section{Authors' Contributions}

Wenxun Liu and Yun Wang contributed equally to this work.

\section{Acknowledgments}

This work was supported by the National Natural Science Foundation of China (no. 81760339); the Fourth Batch of Ningxia Youth Talents Supporting Program; the Ningxia Natural Science Foundation of China (no. 2020AAC03331); and Ningxia Anesthesia Clinical Medical Research Center.

\section{References}

[1] J. Kim, J. P. Villarroel, W. Zhang et al., "The responses of tissues from the brain, heart, kidney, and liver to resuscitation following prolonged cardiac arrest by examining mitochondrial respiration in rats," Oxidative Medicine and Cellular Longevity, vol. 2016, Article ID 7463407, 7 pages, 2016.

[2] H. Li, L. Zhang, Z. Yang et al., "Even four minutes of poor quality of CPR compromises outcome in a porcine model of prolonged cardiac arrest," Biomed Research International, vol. 2013, Article ID 171862, 7 pages, 2013.

[3] J.-Y. Lin, M.-W. Zhang, J.-G. Wang et al., "Hydrogen sulfide improves neural function in rats following cardiopulmonary resuscitation," Experimental and Therapeutic Medicine, vol. 11, no. 2, pp. 577-587, 2016.

[4] H. Jiang, F. Meng, W. Li, L. Tong, H. Qiao, and X. Sun, "Splenectomy ameliorates acute multiple organ damage induced by liver warm ischemia reperfusion in rats," Surgery, vol. 141, no. 1, pp. 32-40, 2007.

[5] Q. Chen, S. Moghaddas, C. L. Hoppel, and E. J. Lesnefsky, "Ischemic defects in the electron transport chain increase the production of reactive oxygen species from isolated rat heart mitochondria," American Journal of Physiology-Cell Physiology, vol. 294, no. 2, pp. C460-C466, 2008.

[6] J. Jiang, X. Fang, Y. Fu, W. Xu, L. Jiang, and Z. Huang, "Impaired cerebral mitochondrial oxidative phosphorylation 
function in a rat model of ventricular fibrillation and cardiopulmonary resuscitation," Biomed Research International, vol. 2014, Article ID 192769, 9 pages, 2014.

[7] T. Kawabe, M. Matsushima, N. Hashimoto, K. Imaizumi, and Y. Hasegawa, "CD40/CD40 ligand interactions in immune responses and pulmonary immunity," Nagoya Journal of Medical Science, vol. 73, no. 3-4, pp. 69-78, 2011.

[8] R. Elgueta, M. J. Benson, V. C. de Vries, A. Wasiuk, Y. Guo, and R. J. Noelle, "Molecular mechanism and function of CD40/CD40L engagement in the immune system," Immunological Reviews, vol. 229, no. 1, pp. 152-172, 2009.

[9] X. Guo, D. Li, M. Chen et al., "miRNA-145 inhibits VSMC proliferation by targeting CD40," Scientific Reports, vol. 6, no. 1, 2016.

[10] X. Guo, L. Yu, M. Chen et al., "miR-145 mediated the role of aspirin in resisting VSMCs proliferation and anti-inflammation through CD40," Journal of Translational Medicine, vol. 14, no. 1, p. 211, 2016.

[11] Z. L. Liu, J. Hu, X. F. Xiao et al., “The CD40 rs1883832 polymorphism affects sepsis susceptibility and SCD40L levels," Biomed Research International, vol. 2018, Article ID 7497314, 8 pages, 2018.

[12] E. N. Benveniste, V. T. Nguyen, and D. R. Wesemann, "Molecular regulation of CD40 gene expression in macrophages and microglia," Brain, Behavior, and Immunity, vol. 18, no. 1, pp. 7-12, 2004.

[13] S. Liachenko, P. Tang, R. L. Hamilton, and Y. Xu, "A reproducible model of circulatory arrest and remote resuscitation in rats for NMR investigation," Stroke, vol. 29, no. 6, pp. 1229-1238, 1998.

[14] G. Paxinos and C. Watson, "The Rat Brain in Stereotaxic Coordinates," Academic Press, San Diego, CA, USA, 4th edition, 1999.

[15] M. Pozzi, C. Koffel, X. Armoiry et al., "Extracorporeal life support for refractory out-of-hospital cardiac arrest: should we still fight for? A single-centre, 5-year experience," International Journal of Cardiology, vol. 204, pp. 70-76, 2016.

[16] X. L. Liu, L. Wiklund, A. Nozari, S. Rubertsson, and S. Basu, "Differences in cerebral reperfusion and oxidative injury after cardiac arrest in pigs," Acta Anaesthesiologica Scandinavica, vol. 47, no. 8, pp. 958-967, 2003.

[17] H. Krep, M. Fischer, and A. Hoeft, "Endothelin-1 elevates regional cerebral perfusion during prolonged ventricular fibrillation cardiac arrest in pigs," Resuscitation, vol. 57, no. 3, pp. 317-318, 2003.

[18] F. Sterz, Y. Leonov, P. Safar, A. Radovsky, S. A. Tisherman, and $\mathrm{K}$. Oku, "Hypertension with or without hemodilution after cardiac arrest in dogs," Stroke, vol. 21, no. 8, pp. 1178-1184, 1990.

[19] P. Safar, W. Behringer, B. W. Bottiger, and F. Sterz, "Cerebral resuscitation potentials for cardiac arrest," Critical Care Medicine, vol. 30, no. 4, pp. S140-S144, 2002.

[20] M. Holzer and F. Sterz, "Therapeutic hypothermia after cardiopulmonary resuscitation," Expert Review of Cardiovascular Therapy, vol. 1, no. 2, pp. 317-325, 2003.

[21] F. Sterz, M. Holzer, R. Roine et al., "Hypothermia after cardiac arrest: a treatment that works," Current Opinion in Critical Care, vol. 9, no. 3, pp. 205-210, 2003.

[22] B. W. Bottiger and E. Martin, "Thrombolytic therapy during cardiopulmonary resuscitation and the role of coagulation activation after cardiac arrest," Current Opinion in Critical Care, vol. 7, no. 3, pp. 176-183, 2001.

[23] C. Antoniades, C. Bakogiannis, D. Tousoulis, A. S. Antonopoulos, and C. Stefanadis, "The CD40/CD40 ligand system: linking inflammation with atherothrombosis," Journal of the American College of Cardiology, vol. 54, no. 8, pp. 669-677, 2009.

[24] B. Zhang, T. Wu, C. Song, M. Chen, H. Li, and R. Guo, "Association of CD40-1C/T polymorphism with cerebral infarction susceptibility and its effect on SCD40L in Chinese population," International Immunopharmacology, vol. 16, no. 4, pp. 461-465, 2013.

[25] X. Ye, W. Zhou, and J. Zhang, "Association of CSF CD40 levels and synaptic degeneration across the alzheimer's disease spectrum," Neuroscience Letters, vol. 694, pp. 41-45, 2019.

[26] S. Gottschling, B. Gronwald, S. Schmitt et al., "Use of complementary and alternative medicine in healthy children and children with chronic medical conditions in Germany," Complementary Therapies in Medicine, vol. 21, no. 1, pp. S61-S69, 2013.

[27] S. C. Lin and A. S. Cheifetz, "The use of complementary and alternative medicine in patients with inflammatory bowel disease," Gastroenterol Hepatol (NY), vol. 14, no. 7, pp. 415425, 2018.

[28] M.-L. Yeh, W.-L. Chiu, Y.-J. Wang, and C. Lo, "An investigation of the use of traditional Chinese medicine and complementary and alternative medicine in stroke patients," Holistic Nursing Practice, vol. 31, no. 6, pp. 400-407, 2017.

[29] A. A. Kadir, A. H. A. Hamid, and M. Mohammad, "Pattern of complementary and alternative medicine use among Malaysian stroke survivors: a hospital-based prospective study," Journal of Traditional and Complementary Medicine, vol. 5, no. 3, pp. 157-160, 2015. 